This item was submitted to Loughborough's Research Repository by the author.

Items in Figshare are protected by copyright, with all rights reserved, unless otherwise indicated.

\title{
An automated target-oriented scanning system for infrastructure applications
}

PLEASE CITE THE PUBLISHED VERSION

https://doi.org/10.1061/9780784482865.049

PUBLISHER

ASCE

VERSION

AM (Accepted Manuscript)

PUBLISHER STATEMENT

This material may be downloaded for personal use only. Any other use requires prior permission of the American Society of Civil Engineers. This material may be found at https://ascelibrary.org/doi/pdf/10.1061/9780784482865.049.

\section{LICENCE}

All Rights Reserved

\section{REPOSITORY RECORD}

Lu, Ruodan, Yuening Ma, Liang Guo, Tony Thorpe, and loannis Brilakis. 2020. "An Automated Target-oriented Scanning System for Infrastructure Applications". Loughborough University.

https://hdl.handle.net/2134/12037590.v1. 


\title{
An automated target-oriented scanning system for infrastructure applications
}

\author{
Ruodan $\mathrm{Lu}^{1,2^{*}}$; Yuening $\mathrm{Ma}^{3}$; Liang $\mathrm{Guo}^{3}$; Tony Thorpe ${ }^{1}$, Ioannis Brilakis ${ }^{4}$
}

${ }^{1}$ School of Architecture, Building and Civil Engineering, Loughborough University, United Kingdom; email: r.lu@ @lboro.ac.uk, t.thorpe@ lboro.ac.uk

${ }^{2}$ Darwin College, University of Cambridge, United Kingdom; email: rl508@ cam.ac.uk

${ }^{3}$ Data Science Institute, School of Mathematics and Statistics, Shandong University, China; email: 201600810419@mail.sdu.edu.cn, liangguo@sdu.edu.cn

${ }^{4}$ Laing O'Rourke Centre, Department of Engineering, University of Cambridge, United Kingdom; email: ib340@cam.ac.uk

\begin{abstract}
Point cloud pre-processing is essential for emerging applications such as digital twinning but currently requires a lot of manual effort before the resulting data can be used. Practitioners usually use default scan range settings to take full scans, which generate huge point cloud datasets containing millions of points. However, only a fraction of the dataset is used for subsequent twinning processes and the remaining data is "noise". Researchers need to perform substantial cropping work to enable the point cloud can be used for detecting objects of interest. However, the problem of object detection in the post-processing stage also remains unresolved. This paper describes a new system TOSS to conduct a target-oriented scanning process. It streamlines the scan-to-gDT (geometric digital twin) process by automatically identifying the region of interest and its corresponding scanning path. TOSS consists of a cost-effective 3DoF rotational laser scanner, a vision-based object detection algorithm, and a geometric-camera-model-based scanning control algorithm. Preliminary results on a real-world bridge indicate that TOSS can produce accurate scans of regions of interest (average: $95.5 \%$ Precision and $89.4 \%$ Recall). It is fully scalable and can be adapted to various infrastructure types, including buildings, bridges, industrial plants, tunnels, and roads. The algorithms also have great potential to be embedded in a traditional scanner's software.
\end{abstract}

\section{INTRODUCTION}

We use the adjective "geometric" to highlight the 3D geometric representation of a digital twin (DT), i.e. gDT. The scan-to-gDT process has become more common as it aims to generate the geometric model of an existing asset using point clouds ( $\mathrm{Lu}$ \& Brilakis, 2019). This process, however, is still far from being fully automatic because processing the scan data is a daunting task. Point cloud pre-processing is an essential step and requires a lot of manual hours before the resulting data can be used for twinning (Lu \& Brilakis, 2019). This is because the attributes of the raw point clouds taken by traditional systems depend fully on the scanning process and the environment of the asset being documented. Practitioners usually use the default scan range setting to take full scans, which generates huge point cloud datasets containing millions of points. Only a fraction of a dataset is used for subsequent twinning processes and the 
remaining data is "noise". Researchers need to perform substantial registering and cropping work before the point cloud can finally be used for detecting objects. The average in-office time spent on registering scans of a typical reinforced concrete bridge was $\sim 10.6$ hours while the manual cropping time was $\sim 1$ hour (Lu et al., 2018).

Leica, Faro, and Trimble are major vendors on the market for the efficient collection of accurate spatial geometry data in the form of point clouds. Each of these vendors provides scanner products that have a specific range scanning function. For example, Leica ScanStation P50/P40/P30 (2019) covers a $360^{\circ} \times 290^{\circ}$ field of view $(\mathrm{FoV})$. The instruments provide three ways to set up a user-defined scan range: 1) designate a custom FoV by setting specific horizontal/vertical (H/V) scan range; 2) manually define the scan area in a 2D bounding box; 3) manually fence an area of interest in the scan viewer screen and add to the scan list as a sub-scan to be re-scanned.

Likewise, Faro Focus 3D X330, M70 and S70/S150/S350 (2019) cover a 360 $\times 300^{\circ} \mathrm{FoV}$. Faro Focus scanners can customize scan areas by setting specific H/V scan range. They also allow users to manually identify scan areas using $2 \mathrm{D}$ bounding boxes. These setting-ups are useful, however, like Leica scanners, they can only draw rectangular areas. In contrast, Trimble SX10 (2019) covers a $360^{\circ} \times 300^{\circ} \mathrm{FoV}$ and allows users to define a customized polygon. This function significantly reduces scanning time and produces a point cloud of region of interest (ROI). Yet, it remains manual and largely depends on an operator's discretion and experience when drawing polygons on a small screen. In summary, existing scanning hardware solutions only provide human-assisted methods to customize specific scan areas.

Usually, the major vendors also provide software packages that are compatible with their scanners, such as Leica Cyclone, Faro Scene, Faro PointSense, Trimble RealWorks, and Trimble EdgeWise. These applications provide solutions for the process of scan-to-gDT in a semi-automatic way. A lot of manual pre-processing is required for removing a huge amount of context points, such as trees, vegetation, traffic, and so on, before the point cloud can be further used for twinning. In this paper, we propose an automated target-oriented scanning process by delivering the ROI and its corresponding scanning path. The proposed scanning process is also embedded into a cost-effective 3-DoF (degree of freedom) rotational laser scanner, which is also part of our innovative system i.e., TOSS.

\section{RESEARCH BACKGROUND}

Numerous detection methods concentrate on generating surface-based primitives of objects of interest. RANdom Sample Consensus (RANSAC) is often used for detecting planar surfaces. Whilst it is effective in the presence of plane outliers, it suffers from spurious planes (Jung et al., 2014), meaning that RANSAC is sensitive around boundaries and tends to generate false positive planes. Walsh et al. (2013) used a Region Growing (RG) algorithm to detect bridge elements in a snippet of a point cloud. However, the RG exhibited boundary weakness so that the segmentation was finally achieved by manually choosing feature points. Likewise, Dimitrov et al. (2016) suggested an RG clustering algorithm to detect building and piping elements in point clouds. However, the point clouds used in the studies were already pre-processed, meaning that irrelevant context points were removed beforehand. Adan \& Huber (2011) proposed Hough-Transform (HT) methods to detect walls in building point 
clouds. However, HT becomes computationally prohibitive when the number of dimensions increases. Xu et al. (2018) suggested an Octree-based (OB) probabilistic segmentation model for construction sites to tackle the computation issue. In general, OB methods are more computationally efficient. However, they are sensitive to the voxel size, which is determined using a user-defined manual approach.

There are also studies discussing the problem of detecting or filtering out unwanted points. Wei et al. (2017) suggested an algorithm to filter out vegetation point clouds collected in the riparian zone covered with multilayer and high-density vegetation. Likewise, Lucas et al. (2019) applied a random forest classifier to separate tall vegetation points from the other points after removing planar surfaces such as grassland and water bodies through scatter feature thresholding. These methods worked well in point clouds of large-scale farmland and mountain areas, where trees or tall vegetations can be distinguished from the surroundings. However, in an urban environment, the vertical features of trees and vegetations might not necessarily be discriminative compared to that of infrastructure.

\subsection{GAPS IN KNOWLEDGE \& OBJECTIVES}

Existing hardware solutions do not provide a fully automatic solution for detecting scan ROI at the pre-processing stage. Depicting boundaries of objects of interest and filtering out unwanted context points in city environments remains unresolved. The objective of this research work is to deliver a novel bespoke scanning system, aiming to automatically conduct a target-oriented scanning process.

\section{PROPOSED SYSTEM}

\subsection{OVERVIEW}

Figure 1 demonstrates the overall workflow of the proposed system TOSS (i.e., target-oriented scanning system). It starts by taking an image using the internal camera followed by sending it via the wireless video transmission module for further processing. A vision-based object detection algorithm is proposed to detect the ROI. Then, we suggest a scanning control algorithm by calculating the angles of each pixel of the ROI relative to the camera. Next, a motion scheme of the motors is suggested followed by performing the target-oriented scanning process. Figure 2 shows the schema of the TOSS scanner system and the hardware prototype. The TOSS scanner is 3-DoF, meaning that it can rotate around 3 axes i.e., roll, yaw, and pitch. It consists of a LiDAR, two wide-angle cameras, and three servo motors. The LiDAR and two cameras are controlled by the microcontroller 1 (MCU 1) while the three servo motors are controlled by the microcontroller 2 (MCU 2). The servo motors and the LiDAR perform the scanning task cooperatively.

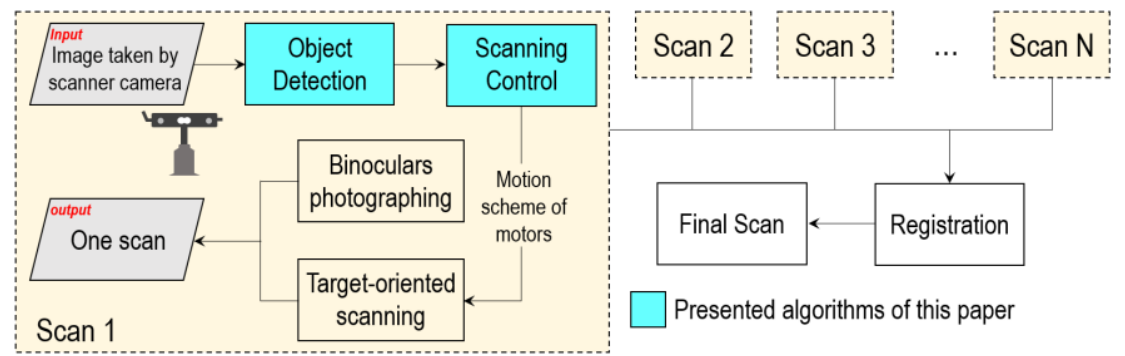

Figure 1. Workflow of the proposed scanning system TOSS 


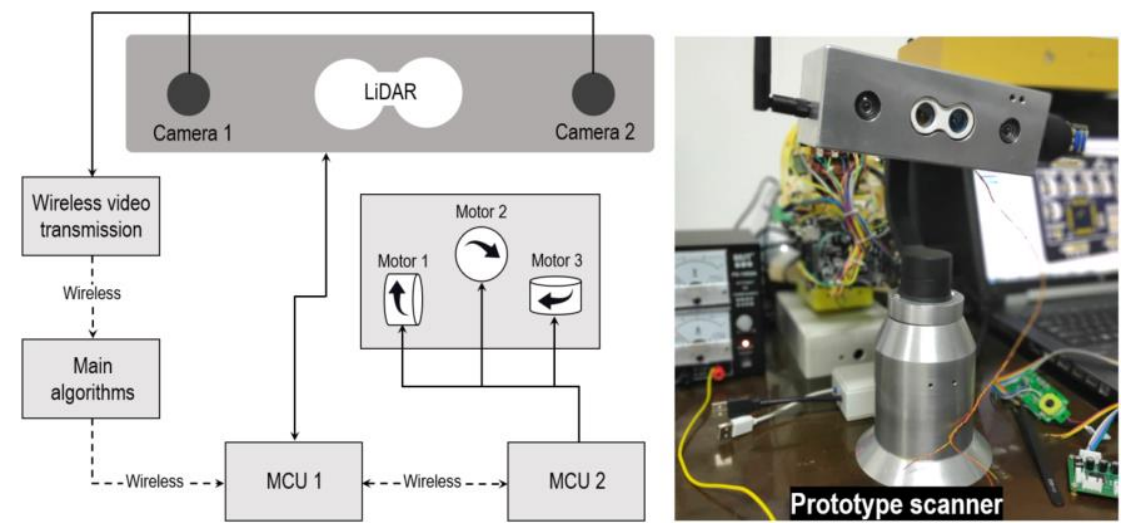

Figure 2. TOSS scanner system

\subsection{OBJECT DETECTION}

Figure 3 illustrates the workflow of the proposed object detection algorithm which consists of six steps. The input is an RGB image $I_{o}$ taken by the scanner internal camera. The final output is the ROI, which is the scan area of the asset of interest in the image. Step 1 starts by reducing the original image size to $X_{r}$-by- $Y_{r}$ in size. Then, Step 2 uses a multi-stage Canny edge detector to detect edges in the resized image $I_{r}$ (Figure 4 (a)). Specifically, we first convert the image $I_{r}$ to grayscale followed by reducing the noise using a Gaussian blur algorithm to smooth it. To do so, a typical image convolution technique is used:

$$
I_{\text {blur }}(i, j)=H_{i j} * I_{r}
$$

where $H_{i j}$ is the Gaussian Kernel of $(2 \mathrm{k}+1)-\mathrm{by}-(2 \mathrm{k}+1)$ in size:

$$
H_{i j}=\frac{1}{2 \pi \sigma^{2}} \exp \left(-\frac{\left(i-(k+1)^{2}+(j-(k+1))\right)^{2}}{2 \sigma^{2}}\right) ; 1 \leq i ; j \leq(2 k+1) \text {. }
$$

The blurred image with a Gaussian filter is then used to compute the gradient magnitude followed by applying the non-maxima suppression to the gradient magnitude image. Next, the detector uses the double thresholding and edge tracking to detect strong edges. We then acquire a binary image with white edges $I_{r_{-} \text {bin }}$ of the asset being documented. Next, Step 3 uses HT to detect line segments (denoted as $S_{s t r}$ ) in the resulting binary image $I_{r_{-} b i n}$.

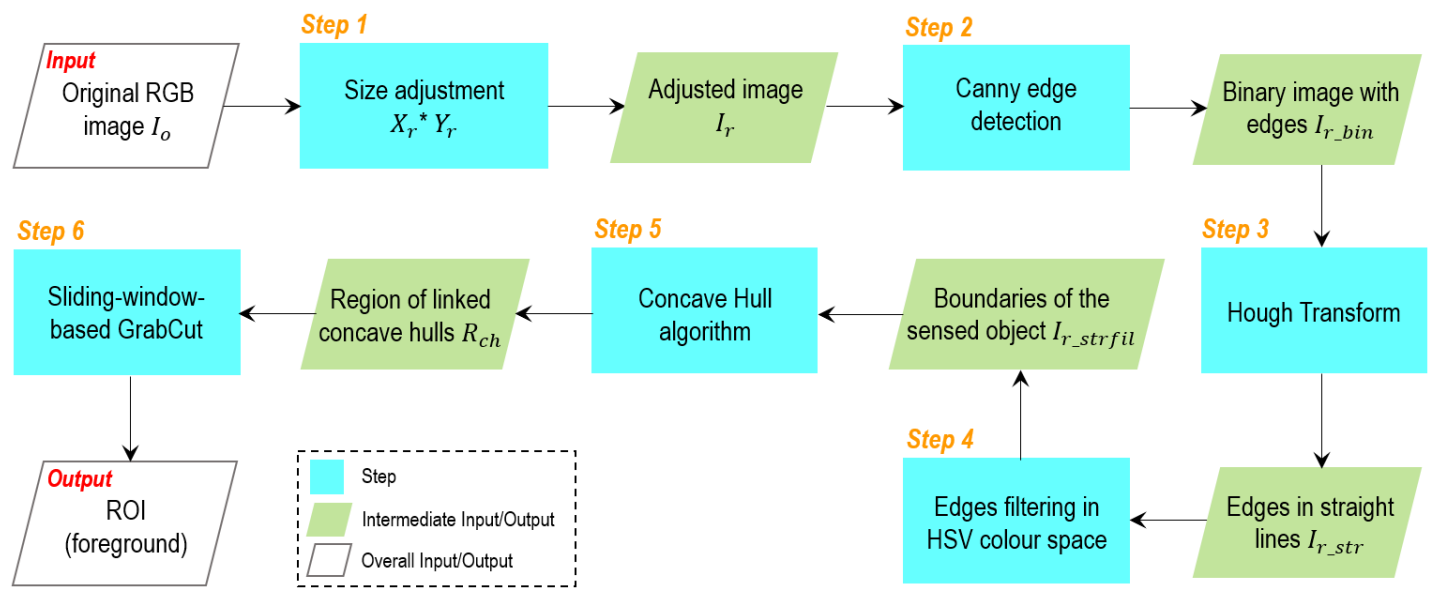

Figure 3. Workflow of the detection algorithm 
Some infrastructure may contain curved elements. However, the curvature of sine-like elements, such as the deck slabs of typical highway bridges stays within a certain degree. HT detects all possible line segments including many misclassified environment ones. Figure 4 (b) shows an example that many line segments are detected as false positives in abutment, ramp, and vegetations areas of a synthetic bridge scene. We divide the image boundaries into multiple line segments with a length $l_{\text {thred }}$ in order to handle the problem that some interesting parts may be outside the FoV of the camera. Hence, Step 4 intends to filter out wrongly detected edges i.e., environment line segments in the image $I_{r_{-} s t r}$ generated from Step 3. This is achieved using the contrast of hue to create distinct areas of the infrastructure and the background. The problem of filtering out false-positive edges is transformed into a binary classification problem. We introduce the concept of local homogeneity into a Hue-value-histogrambased algorithm. We first convert the RGB-based image $I_{r}$ into HSV (Hue, Saturation and Value)-based image $I_{r_{-} H S V}$. Then, $\forall s \in S_{s t r}$, we consider its neighbouring pixels within a region of size $\sim 2 d * l_{s t r}$, where $l_{s t r}$ is the length of $s$, as a local homogeneity region (LHR). We calculate the Hue-value of every pixel in each half-LHR $\left(d * l_{s t r}\right)$ followed by using histograms to find its dominant Hue-value. Each line segment $s$ has a pair of dominant Hue-value, denoted as $H_{s_{1}}, H_{s_{2}}$. Once the pair-wise dominant Huevalue of each line segment $s$ is determined, we can cluster the Hue-values of $S_{s t r}$ into the asset of interest and the environment regions using the histogram again based-on different Hue-value ranges. The Hue-value of the infrastructure $H_{\text {infra }}$ is deemed as the central value corresponding to its maximum bin: if $\forall s \in S_{s t r}$, and $H_{s_{i}}=H_{\text {infra }}, i=$ 1,2 , then, $s \in I n f r a$; otherwise, $s \in$ environment. This way, the false-positive environment line segments are filtered out. We acquire an image $I_{r_{-} s t r f i l}$ with only line segments belonging to the infrastructure (denoted as $S_{\text {infra }}$ ). Step 5 tends to link the lines segments $S_{\text {infra }}$ using a 2D ConcaveHull $\alpha$-shape. Only the two extremities of $S_{\text {infra }}$ are used to compute the hulls. Figure 5 illustrates the region of linked concave hulls $R_{c h}$ of the extremity pixels of lines segments $S_{\text {infra }}$. Some bridge areas (i.e., $R_{M}$ ) are enclosed outside the boundaries due to the concave nature of the $\alpha$-shape algorithm while some areas in $R_{c h}$ are the environment, i.e., $R_{E}$. We need a technique that can flexibly add the missing regions $R_{M}$ and remove the regions of environment $R_{E}$. Therefore, Step 6 proposes a sliding-window-based GrabCut algorithm, aiming to tackle the abovementioned segmentation challenge. GrabCut is an iterative technique built upon Graph Cut. We define the foreground as the region based on the concave hulls $R_{c h}$. Specifically, we first create an image mask by assigning 4 class labels $\alpha$ to image pixels $\{p\}$, i.e., $T_{B}$ : Background $\leftarrow \alpha_{p}=0, T_{1}$ : Suspected background $\leftarrow \alpha_{p}=1$, $T_{2}$ : suspected foreground $\leftarrow \alpha_{p}=2, R_{c h}:$ Foreground $\leftarrow \alpha_{p}=3$ using the dilation technique: $R_{c h} \oplus d_{j}$, where $d_{j}$ is a structuring element (Figure 6). We detect the environment regions $R_{E}$ that are inside the $R_{c h}$ by re-assigning their labels using a sliding window technique. The label of the area of the window $A_{w}$ stays the same if the latter does not overlap with $R_{c h}$; otherwise, it will be re-assigned a new label (i.e., $\alpha_{p}=1$ for $p \in A_{w}$ ). Two Gaussian mixture Models (GMMs) with $K$ components are created, one for the classes of background and the suspected background, and the other one for the classes of the foreground and the suspected foreground. 


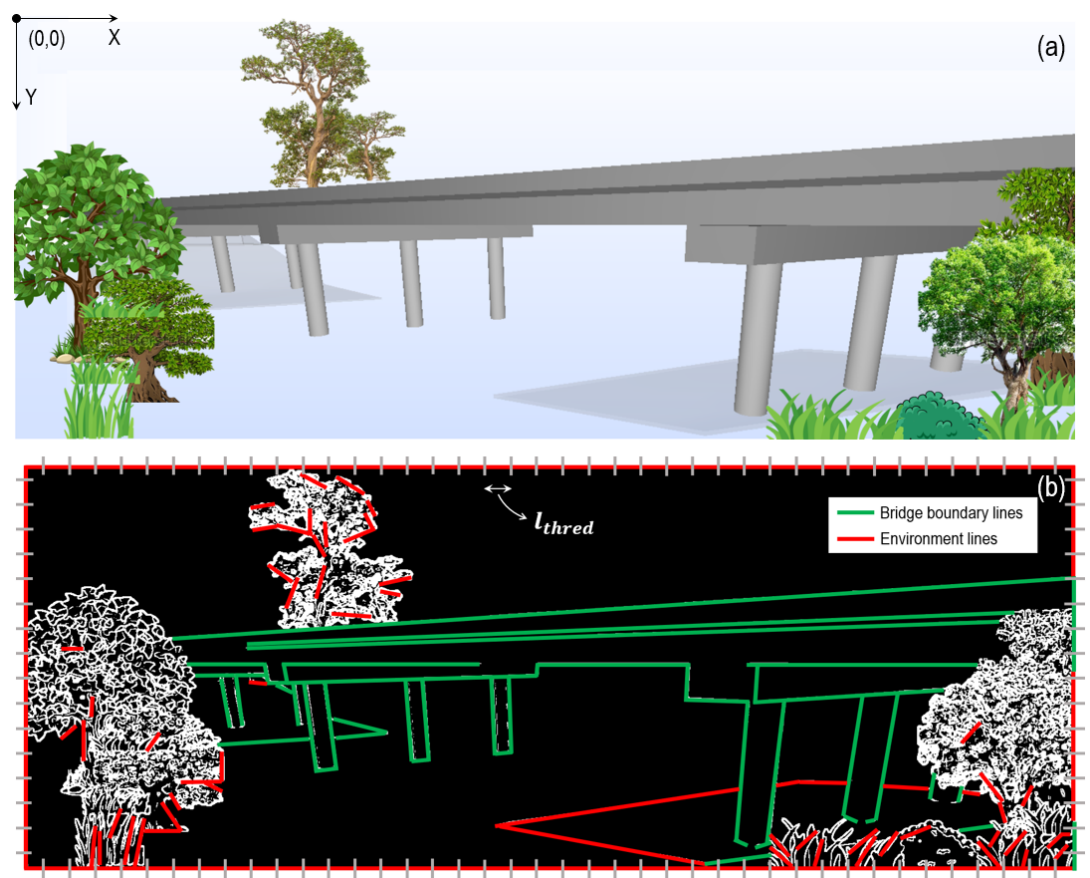

Figure 4. A synthetic bridge scene being documented: (a) Adjusted RGB image $I_{r} ;$ (b) Line segments $S_{\text {str }}$ detected in $I_{r_{-} b i n}$

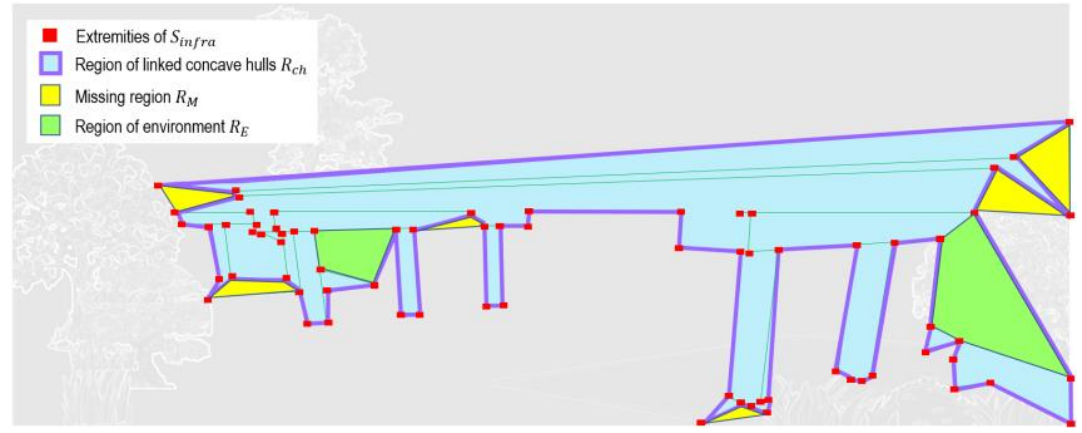

Figure 5. Linked concave hulls of the synthetic bridge

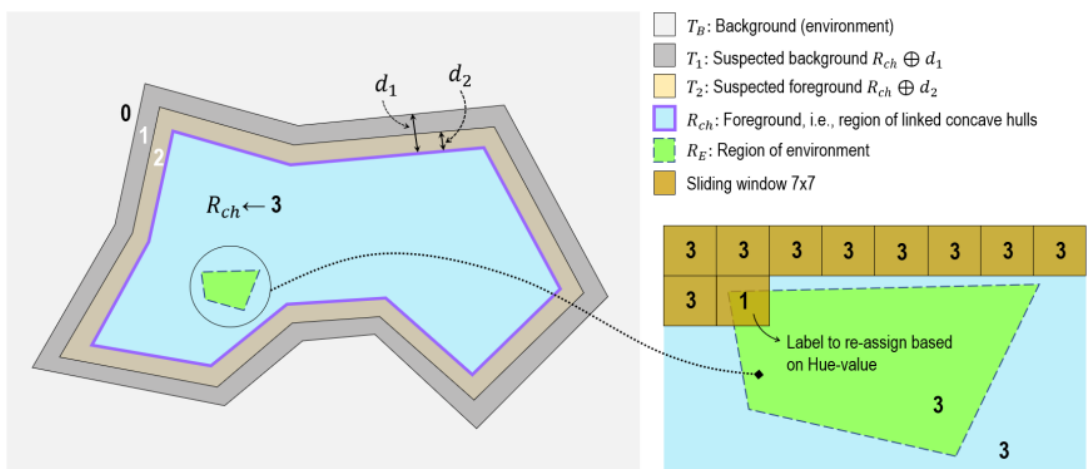

Figure 6. Labelled image mask and sliding window to detect $\mathbf{R}_{E}$

Then, $\forall p \in T_{B}$ and $p \in T_{1}$ (i.e., $\alpha_{p}=\{0,1\}$ ), $p$ is assigned to the most likely Gaussian component $k_{n}$ for $k_{n} \in\{1,2, \ldots, K\}$ in the background GMM whilst $\forall p \in T_{2}$ and $p \in$ 
$R_{c h}$ (i.e., $\left.\alpha_{p}=\{2,3\}\right), p$ is assigned to the most likely foreground Gaussian component. Updated GMMs are learnt from the pixel sets created in the previous sets followed by minimizing the Gibbs energy function for the suspected regions (i.e., $T_{1+2}$ ) to conduct the Graph Cut. The process starting from the GMM component assignment step is iterated until the classification convergence and the ROI of the image is yielded.

\subsection{SCANNING CONTROL}

This section aims to control the motion scheme of the TOSS motors for the LiDAR to perform the scanning process based on the ROI. The scanning control is the problem of controlling the rotational angles. It consists of two major processes: Process (1) - calculate the angles of the real-world coordinates corresponding to the pixels in the scan area relative to the LiDAR, and Process (2) - plan the motion of two servo motors that are responsible for scanning and produce the command sequence for motor rotation. First, the scan area is the binary image containing the ROI resulting from the previous step plus the margin areas, i.e., $R O I_{\text {scan }}=\mathrm{ROI} \oplus \mathrm{m}$. Then, the image of $R O I_{\text {scan }}$ is re-sized into its original size (denoted as $I_{R O I_{s c a n}}$ ). We assume the angles (the altitude and the azimuth) of a given pixel of the ROI relative to the LiDAR is approximately equal to the altitude and the azimuth relative to the camera because the LiDAR is very close to the camera $(<10 \mathrm{~cm})$. Given a point $P$ on the surface of the infrastructure being documented, it can be represented in different homogenous coordinates: $p_{\text {pix }}\left(x_{p_{p i x}}, y_{p_{\text {pix }}}, 1\right)$ for $P$ in the image plane, $\hat{p}\left(x_{\hat{p}}, y_{\hat{p}}, 1\right)$ and $\hat{p}^{\prime}\left(x_{\hat{p}^{\prime}}, y_{\hat{p}^{\prime}}, 1\right)$ for $P$ in the normalized planes, and $P_{C}\left(x_{P_{C}}, y_{P_{C}}, z_{P_{C}}, 1\right)$ for $P$ in the 3D camera system. According to the geometric relationships of the camera imaging system (Figure 7 (a)), the normalized planes $\hat{\pi}$ and $\hat{\pi}^{\prime}$ and their corresponding coordinate systems are centrosymmetric so that $\hat{p}^{\prime}=\hat{p}$. The azimuth (denoted as $\theta_{x}$ ) and the altitude (denoted as $\theta_{y}$ ) relative to the camera principal point of $O$ for both $P_{C}$ and $\hat{p}^{\prime}$ are the same. Therefore, the problem of Process (1) is transformed into deriving $\theta_{x}$ and $\theta_{y}$ relative to $O$ in the camera coordinate system (Figure $7(\mathrm{~b})$ ):

$$
\begin{array}{ll}
\theta_{x}=\arctan \frac{x_{\hat{p}^{\prime}}}{1 m m}=\arctan x_{\hat{p}^{\prime}}, & \text { Eq.3 } \\
\theta_{y}=\arctan \frac{-y_{\hat{p}^{\prime}}}{1 m m}=-\arctan y_{\hat{p}^{\prime}} . & \text { Eq.4 }
\end{array}
$$

We define the pixel coordinates in the normalized plane using:

$$
\hat{p}=K^{-1} p_{\text {pix }} \text {, }
$$

where $K$ is the internal calibration matrix of the camera. We propose a multi-step method, consisting four phases to derive $\hat{p}$ from $p_{\text {pix }}$. First, we reserve some space for the image shearing by extending $I_{R O I_{s c a n}}$ to the left and right sides by $q$ black pixels. That is, given $I_{R O I_{s c a n}}$ is $m$-by- $n$ in size, then the size of $I_{R O I_{s c a n}}$ becomes $m$-by- $(n+2 q)$. A given pixel $p_{\text {pix }}\left(x_{\text {pix }}, y_{\text {pix }}, 1\right)$ of $I_{R O I_{s c a n}}$ becomes:

$p_{1}=p_{\text {pix }}+(q, 0,0)$.

Next, we only apply shearing and scaling to $p_{1}$ :

$$
p_{2}=\left(\begin{array}{ccc}
1 & \cot \theta & 0 \\
0 & 1 & 0 \\
0 & 0 & 1
\end{array}\right)\left(\begin{array}{ccc}
\frac{1}{\alpha} & 0 & 0 \\
0 & \frac{\sin \theta}{\beta} & 0 \\
0 & 0 & 1
\end{array}\right)\left(p_{p i x}+(q, 0,0)\right) .
$$




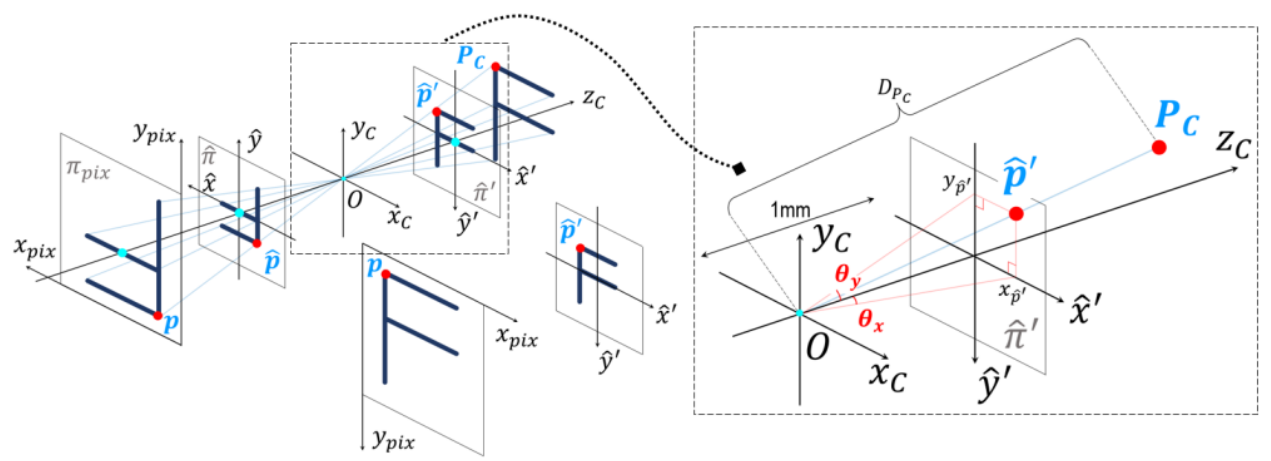

(a)

(b)

Figure 7. Geometric model of the camera imaging principle

The image is then transformed into a parallelogram $I_{\widetilde{R O} I_{s c a n}}$ followed by multiplying by $Z$ and rounding it:

$$
p_{3}=\operatorname{round}\left(\left(\begin{array}{ccc}
Z & 0 & 0 \\
0 & Z & 0 \\
0 & 0 & 1
\end{array}\right) p_{2}\right) \text {. }
$$

An empty matrix of size $\left\|Z(n+2 q) \cdot \frac{1}{\alpha}\right\| *\left\|Z m \cdot \frac{\sin \theta}{\beta}\right\|$ is used to store $I_{R \widetilde{R O} I_{s c a n}}$. We discard the area exceeding the matrix while the missing area is filled in black (Figure 8). The azimuth and the altitude can be deduced:

$$
\begin{aligned}
& \theta_{x}=\arctan x_{\hat{p}^{\prime}} \approx \arctan \left(\frac{x_{p_{3}}}{Z}-\left(\frac{q}{\alpha}+\frac{x_{0}}{\alpha}+\frac{y_{0} \cdot \cos \theta}{\beta}\right)\right), \\
& \theta_{y}=\arctan y_{\hat{p}^{\prime}} \approx \arctan \left(\frac{y_{p_{3}}}{Z}-\frac{y_{0} \cdot \sin \theta}{\beta}\right) .
\end{aligned}
$$

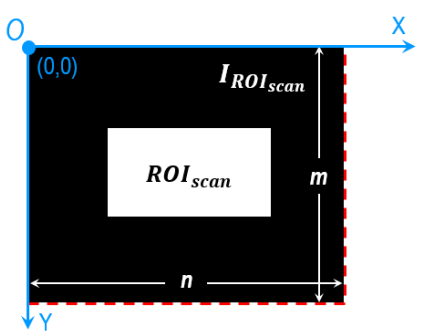

(a) Image with updated scan area after dilation $\left(\mathrm{I}_{\mathrm{ROI}_{\text {scan }}}\right)$

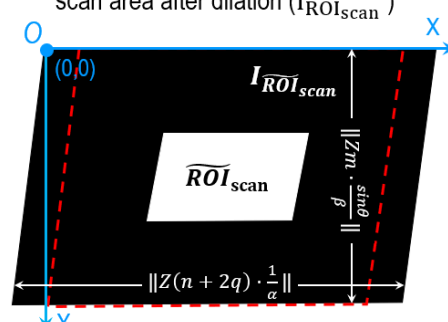

(d) $\mathrm{I}_{\mathrm{ROI}^{\prime}}{ }_{\text {scan }}$ after scaling and multiplying $\mathrm{Z}\left(\mathrm{I}_{\widetilde{\mathrm{RO}}} \mathrm{I}_{\text {scan }}\right)$

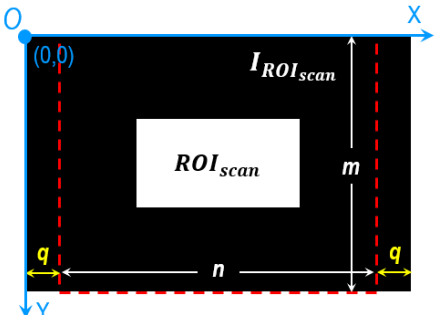

(b) $\mathrm{I}_{\mathrm{ROI}_{\text {scan }}}$ with extended two borders

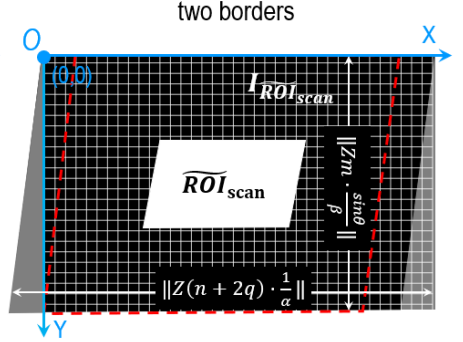

(e) Save the transformed image

$\left(\mathrm{I}_{\mathrm{ROI}_{\text {scan }}}\right)$

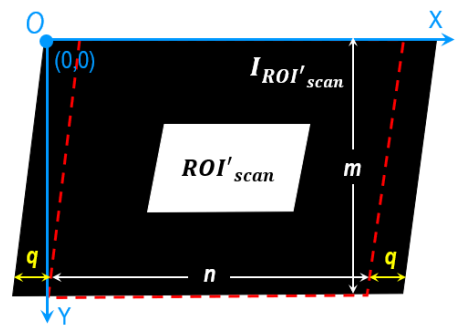

(c) $\mathrm{I}_{\mathrm{ROI}_{\text {scan }}}$ after shearing $\left(\mathrm{I}_{\mathrm{ROI}^{\prime}{ }_{\text {scan }}}\right)$

Boundaries of $\mathrm{I}_{\mathrm{ROI}_{\text {scan }}}$ $L_{-}{ }_{-}$Boundaries of $\mathrm{I}_{\mathrm{ROI}_{\text {scan }}}$ after shearing Areas to discard or fill in black

Figure 8. Multi-step coordinates transformation 
Once we acquire $I_{\widetilde{R O}} I_{s c a n}$ and pixels along with the corresponding azimuths and altitudes, we can plan the motion scheme i.e., the scanning path. We propose a threestep controlling algorithm that suggests an " $S$ " shaped scanning path by column. Specifically, Step 1 deduces the minimum $\left(\theta_{x_{\text {start }}}\right)$ and maximum azimuth $\left(\theta_{x_{\text {end }}}\right)$ based on the minimum $\left(x_{\min }\right)$ and maximum $\left(x_{\max }\right)$ horizontal coordinates in $\widetilde{R O} I_{\text {scan }}$ followed by generating the sequence $\Theta_{x}$. Then, Step 2 deduces $\theta_{y_{\text {start }}}, \theta_{y_{\text {end }}}$ and the sequence $\Theta_{y}$ in a similar way. Step 3 calculates and records $P_{C}\left(x_{P_{C}}, y_{P_{C}}, z_{P_{C}}\right)$ if $\left(x_{i}, y_{j}\right)$ is inside $\widetilde{R O} I_{\text {scan }}$; otherwise, no action is required.

\section{EXPERIMENTS AND PRELIMINARY RESULTS}

Our experiments consist of two parts: object detection and scanning control. We only demonstrate the first part in this paper due to limited space. We used the internal camera to collect 20 images from different standpoints around a real-world concrete highway bridge (GPS: 121.686563,37.439334). The images were adjusted into size of $900 * 600$. The performance of the algorithm was evaluated using Recall and Precision, which are defined as:

$$
\text { Recall }=\frac{\text { True Positives }}{\text { True Positives+False Negatives }}, \quad \text { Eq.11 } \quad \text { Precision }=\frac{\text { True Positives }}{\text { True Positives+False Positives }} . \quad \text { Eq.12 }
$$
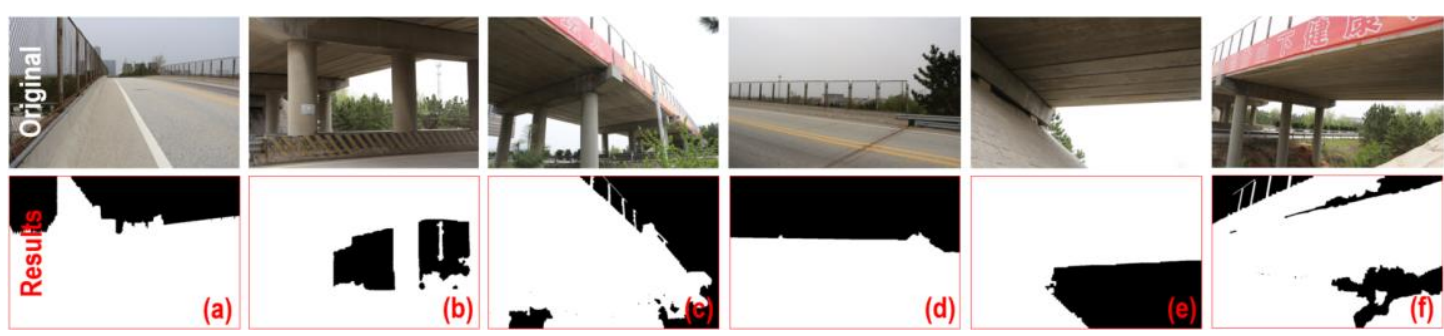

Figure 9. Experiments on real-world bridge images

Table 1 Performance - Recall, Precision and ROI (\%)

\begin{tabular}{lcccccc}
\hline Image & a & b & c & d & e & f \\
\hline Recall & $90.2 \%$ & $99.7 \%$ & $94.9 \%$ & $100 \%$ & $99.7 \%$ & $87.1 \%$ \\
Precision & $97.6 \%$ & $96.1 \%$ & $79.4 \%$ & $90.1 \%$ & $99.7 \%$ & $72.0 \%$ \\
ROI/all & $72.7 \%$ & $83.5 \%$ & $70.9 \%$ & $58.3 \%$ & $74.2 \%$ & $80.4 \%$ \\
\hline
\end{tabular}

Figure 9 and Table 1 show part of the detection results due to limited space. On average, we had $95.5 \%$ Recall, $89.4 \%$ Precision, and $73.0 \%$ flagged ROI rate. Almost all the ROIs in these images were correctly extracted. Undetected ROIs were mainly concentrated in the handrail areas at boundaries. The performance of the image (f) was affected due to the on-site occlusions of the large banner on the handrails. The banner is red whose Hue-value is close to that of the sky. The vegetations underneath the bridge where the lighting is poor also affected the performance.

\section{CONCLUSION}

We present TOSS, a bespoke scanning system that can conduct a target-oriented scanning process. Preliminary results on a real-world bridge demonstrate its promising detection performance. More detailed results will be published later. TOSS is the first 
system of its kind to provide a proactive scanning solution to produce ROI of an asset being documented. It has great potential to be adapted to different infrastructure types and to apply to any combination of camera and scanner solution.

\section{REFERENCES}

Adan, A., \& Huber, D. (2011). 3D reconstruction of interior wall surfaces under occlusion and clutter. Proceedings - 3DIMPVT 2011, 275-281. https://doi.org/10.1109/3DIMPVT.2011.42

Dimitrov, A., Gu, R., \& Golparvar-Fard, M. (2016). Non-Uniform B-Spline Surface Fitting from Unordered 3D Point Clouds for As-Built Modeling. CACAIE, 31(7), 483-498. https://doi.org/10.1111/mice.12192

Faro Focus. (2019). Available at https://www.faro.com/en-gb/products/constructionbim-cim/faro-focus/. Last Accessed 25 July 2019.

Jung, J., Hong, S., Jeong, S., Kim, S., Cho, H., Hong, S., \& Heo, J. (2014). Productive modeling for development of as-built BIM of existing indoor structures. Autom. Constr, 42, 68-77. https://doi.org/10.1016/j.autcon.2014.02.021

Leica scanners. (2019). Available at https://leica-geosystems.com/products/laserscanners/scanners. Last Accessed 25 July 2019.

Lu, R., Brilakis, I., Middleton, C.R. (2018). Detection of structural components in point clouds of existing RC bridges. CACAIE. https://doi.org/10.1111/mice.12407

Lu, R., \& Brilakis, I. (2019). Digital twinning of existing reinforced concrete bridges from labelled point clusters. Autom. Constr, https://doi.org/10.1016/j.autcon.2019.102837

Lucas, C., Bouten, W., Koma, Z., Kissling, W. D., \& Seijmonsbergen, A. C. (2019). Identification of linear vegetation elements in a rural landscape using LiDAR point clouds. Remote Sensing. https://doi.org/10.3390/rs11030292

Trimble SX10. (2019). Available at https://geospatial.trimble.com/SX10_stories. Last Accessed 25 July 2019.

Walsh, S. B., Borello, D. J., Guldur, B., \& Hajjar, J. F. (2013). Data Processing of Point Clouds for Object Detection for Structural Engineering Applications. CACAIE, 28(7), 495-508. https://doi.org/10.1111/mice.12016

Wei, L., Yang, B., Jiang, J., Cao, G., \& Wu, M. (2017). Vegetation filtering algorithm for UAV-borne lidar point clouds: a case study in the middle-lower Yangtze River riparian zone. International Journal of Remote Sensing. https://doi.org/10.1080/01431161.2016.1252476

$\mathrm{Xu}$, Y., Tuttas, S., Hoegner, L., \& Stilla, U. (2018). Voxel-based segmentation of 3D point clouds from construction sites using a probabilistic connectivity model. Pattern Recognition Letters, 102, 67-74. https://doi.org/10.1016/j.patrec.2017.12.016 